\title{
Computer Modeling of Aerosol Particle Transport through Lung Mucosa
}

\author{
Blake Bartlett ${ }^{1}$, Yu Feng ${ }^{1}$, Catherine A. Fromen ${ }^{2}$, and Ashlee N. \\ Ford Versypt ${ }^{1,3}$ \\ ${ }^{1}$ Oklahoma State University \\ ${ }^{2}$ University of Delaware \\ ${ }^{3}$ University at Buffalo, The State University of New York
}

October 18, 2021

\begin{abstract}
Delivery of aerosols to the lungs have great potential for the treatment of various lung diseases. However, the lungs are coated by a protective mucus layer whose complex properties make this form of delivery difficult. Mucus is non-Newtonian and cleared from the lungs over time by ciliated cells. Further, its gel-like structure hinders the diffusion of particles through it. Any aerosolized lung disease treatment must have certain properties to circumvent this barrier, and these properties may vary between diseases, drugs, and patients. Using Computational Fluid Dynamics (CFD) modeling, a model of this mucus layer was constructed such that the diffusion of an impacted aerosol might be studied. The model predicts what amount of a particle of a certain size might be expected to penetrate the mucus and reach the underlying tissue, as well as the distance downstream of the dosage site where concentration is maximized. Using this information, a personalized treatment plan may be designed. The model maintains modularity so that various lung regions and patient health states may be simulated.
\end{abstract}

\section{Introduction}

Lung diseases afflict hundreds of millions of people and are some of the most common causes of death worldwide. Existing methods of treating these diseases are limited by poor targeting to the lungs when medications are given orally or intravenously and require rigorous, long-term, often invasive medication to achieve remission in pathogenic disease $[1,2]$. Other diseases are chronic but suffer similar limitations in treatment methods, inhaled or otherwise. The idea of treating lung diseases at the source is an attractive one, offering a noninvasive route to locally dose a diseased area that also minimizes side effects; 
however, several problems still exist. One specific example is the mucociliary clearance (MCC) mechanism, which utilizes rhythmically waving ciliated cells to constantly push the mucus layer that coats the lungs upward towards the throat. This system serves as a natural defense against infection and particle buildup but simultaneously acts as a barrier to drug delivery.

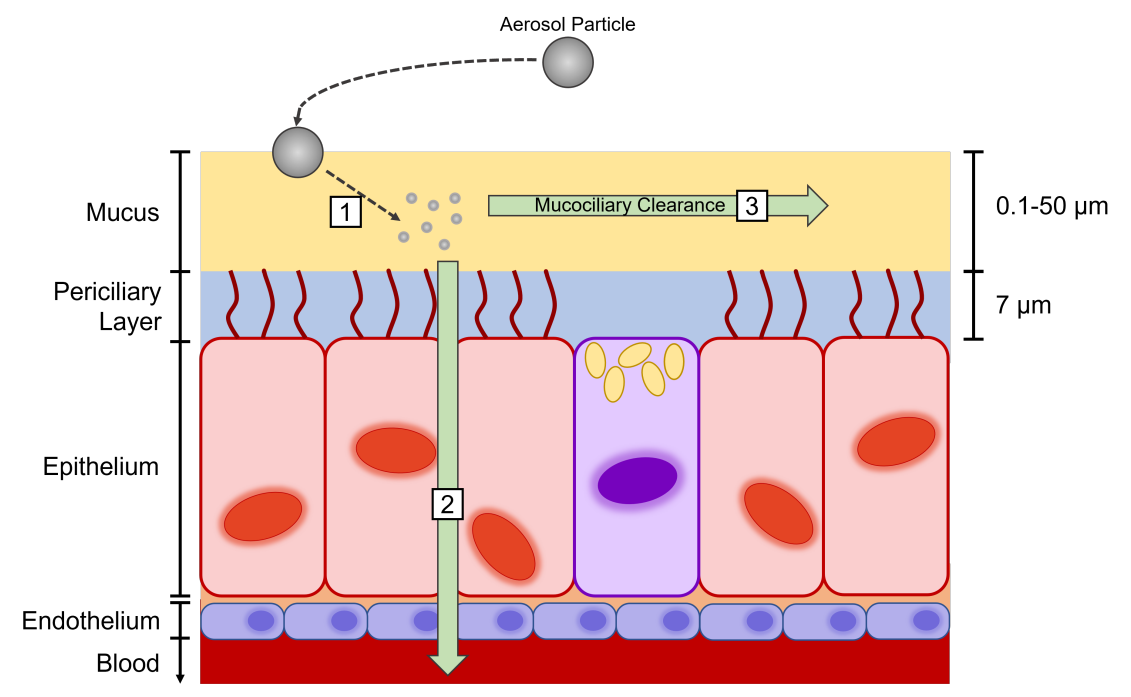

Figure 1: Methods of clearance from the mucosa. (1) Particles can be degraded or consumed by macrophages. (2) Particles can successfully diffuse across the mucosa and be taken up into the tissue or even the bloodstream. (3) Most commonly particles are either unable to diffuse or are immobilized by some means and cleared by MCC.

The anatomical interface between the airways and the lung epithelium is comprised of a fluid bilayer, with the highly viscous mucus layer on top of the more watery periciliary layer $[3,4]$ (see Fig. 1). The periciliary layer allows the cilia to beat without catching in the mucus and acts as a lubricant that allows the mucus to slide along the interface $[5,6]$. The mucus itself is mostly water, but a network of glycoproteins called mucins causes it to move as a sheet when pushed by the cilia. Mucins are rich in cysteine, making them largely anionic [6]. Disulfide bonds are a large contributor to the structure of the network. However, large portions of the chains are neutral, making mucins also appreciably lipophilic [7]. The periciliary layer also contains mucins, but these are tethered to cilia and do not form the tangled net that is seen in the mucus layer. This makes the periciliary layer a "brush" that prevents mixing of the two layers and maintains the viscous character of the periciliary layer compared to the more elastic character of the mucus layer [8]. Because the cilia are regular in size, the periciliary layer is consistently $\approx 7 \mu \mathrm{m}$ thick through the entire tracheobronchial tree [3]. In contrast, the mucus layer varies signif- 
icantly depending on location. Goblet cells, which secrete mucus, are present throughout the respiratory tract and are more plentiful in the upper airways [9]. Thus, the mucus layer increases in thickness from the lower airways to the upper (see Fig. 2). Mucus can be as thin as $0.5 \mu \mathrm{m}$ at the alveolar level (at the deepest levels of the respiratory tract) and as thick as $100 \mu \mathrm{m}$ at the trachea $[4,5]$. The velocity at which mucus is cleared depends on a variety of factors that vary significantly between individuals including age, health, and history of smoking. The tangled network of mucins in the mucus layer gives the layer

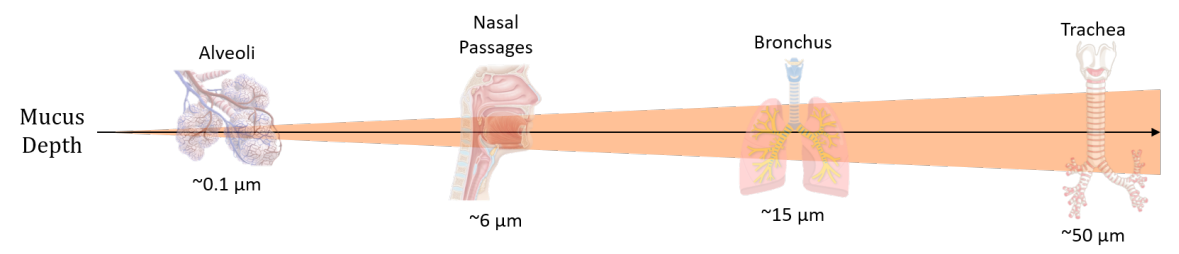

Figure 2: Mucus layer depth thins toward more distal regions of the lung. As the nasal passages are also a mucosal tissue, an average value for mucus depth there is also given for comparison to the regions of the tracheobronchial tree in the lungs. Values given are estimates for a healthy individual.

marked elastic properties, behaving as a viscoelastic gel, which for our purposes is approximated as a shear-thinning non-Newtonian fluid $[7,10,11,12]$. This relationship exists in healthy individuals (and is the reason coughing is an effective method of clearing mucus), but is accentuated in obstructive lung diseases like cystic fibrosis (CF), chronic obstructive pulmonary disease (COPD), and asthma. In these diseases, mucin production is elevated, causing the mucus to become much thicker and more difficult to clear than in the healthy case [13]. Using a non-Newtonian mathematical model for the mucus allows for a single simulation that can be applied to both healthy and diseased states without the need for tables of viscosity measurements.

The rheological properties of lung mucus must be taken into account to successfully treat lung disease via an inhaled aerosol, and the drug particles must have certain properties to pass through the mucus quickly and efficiently. A model of these fluid layers can predict the effectiveness of a delivered pharmaceutical or be modified to simulate the infectivity of a pathogen. While researchers have been optimistic about the possibility of aerosol treatments for various lung conditions and diseases for decades $[14,15,16]$, poor characterization of the mucosa has been a stumbling block for progress. Developments in the production of nanoparticles, aerosolization, and delivery to specific sites in the lung have made these treatments much more attainable $[17,18]$, but the literature lacks a generalized model for predicting behavior in the mucus. Models that exist largely focus on cilial beating $[12,19]$, individual pores of the mucus layer [20,21,22], or are simplified to the point of ignoring fluid movement or the existence of multiple fluid layers [23, 24], which leave questions about locating dosage sites or macroscopic behavior unanswered. The purpose of this 
research is to help fill that gap by producing a model that simulates the mucosa as a whole and predicts the behavior of an applied aerosolized particle. In this paper, we construct a computational fluid dynamics model of the lung mucosa, accomplished using data taken from the literature.

\section{Methods}

\subsection{COMSOL Modeling}

The domain of the model is two-dimensional, consisting of a series of four rectangles, arranged in quadrants (Figs. 3-9). Where the edges of these rectangles meet, COMSOL automatically forms a union between them, such that there is no formal boundary condition separating them. The model domain represents a cross-section of the mucosa at any particular location in the tracheobronchial tree (or nasal passages), and it is assumed that the tracheobronchial tree is radially symmetric. In terms of the biological system, the top two rectangles represent the mucus layer, and the bottom two rectangles represent the underlying periciliary layer. The rectangles on the left have the same fluid properties as the rectangles on the right with respect to their layer, but the left side functions as the dosage site, whereas the right side represents fluid upstream of this site. There is no need to expand the domain to the left of the dosage site as fluid convective velocity is several orders of magnitude higher than diffusive velocity, so backflow is negligible. The height of the model is dependent on the lung location being simulated (Fig. 2), but is always on the scale of microns, so all geometrical dimensions for the model are on this scale. The depth of the periciliary (lower) layer is effectively constant throughout the tracheobronchial tree at $7 \mu \mathrm{m}$. The length of the tracheobronchial tree is on the scale of decimeters [5], so the width of the model is set arbitrarily as we would not expect a marked change in mucus depth over any of the comparatively small widths simulated in this paper. All simulation domains shown in this paper have a total width of $80 \mu \mathrm{m}$.

The upper boundary of the mucus is open to the airways, making it a free surface under general conditions, and is governed by a slip condition. Conversely, the lower boundary of the periciliary layer borders the static epithelium, and is subject to a no slip condition. Bulk fluid moves from the left boundary of both layers to an outlet at the right boundaries without accumulation within the simulation domain. High viscosity and low velocity means that the mucus has a small Reynolds number, moving in a laminar flow regime. For the simulations shown in this paper, the dilute species enters from the airways at a constant concentration constraint, which makes steady-state simulations possible. The dilute species may exit at the rightmost boundary, simulating convective clearance by MCC, or at the bottom boundary, simulating uptake into the epithelium (which is assumed to occur instantaneously when the solute reaches the bottom boundary). This behavior is accomplished using outflow boundary conditions on these edges. The dilute species cannot cross the upper boundary, as this would 


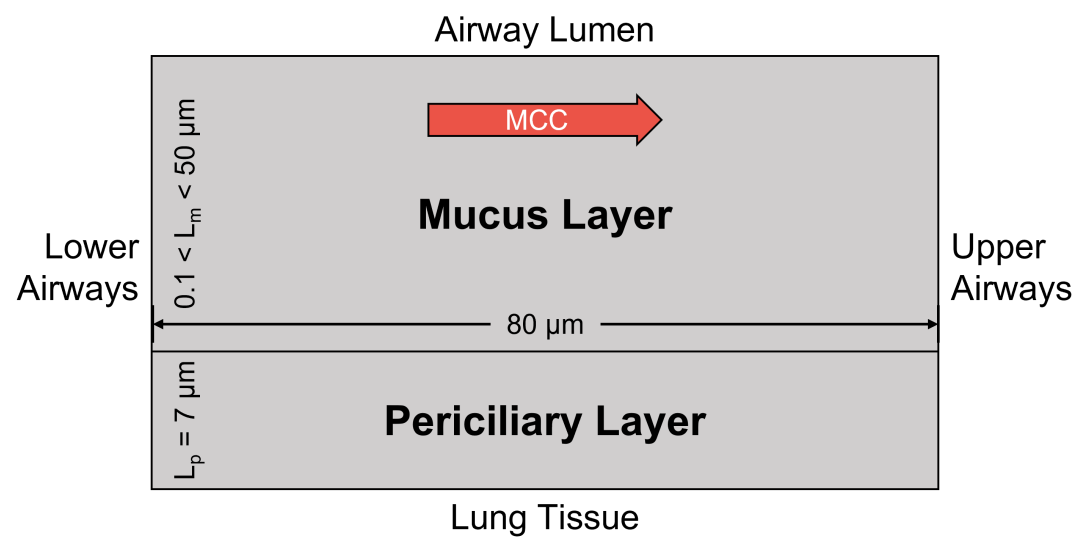

Figure 3: Example of geometry used in model. The top layer is the mucus and is open to the conducting airways, also called the lumen. The bottom layer is the periciliary layer; the cilia beat through this layer and are attached to cells of the lung epithelium. Mucus flows from the lower airways to the upper airways and the throat, and this directionality is simulated as left to right in the diagram.

imply that the applied nanoparticle may freely vaporize. There is nothing preventing the dilute species from crossing the leftmost boundary, and as such is given an outflow boundary condition, but in practice backflow does not occur due to the differences in magnitude between convective and diffusive velocities.

A mesh is automatically generated by COMSOL Multiphysics for the model. This triangular mesh provides discrete points at which each relevant system of equations is solved resulting in a two-dimensional field of behavior across the simulation domain. The mesh may be refined manually at the price of increased computation times.

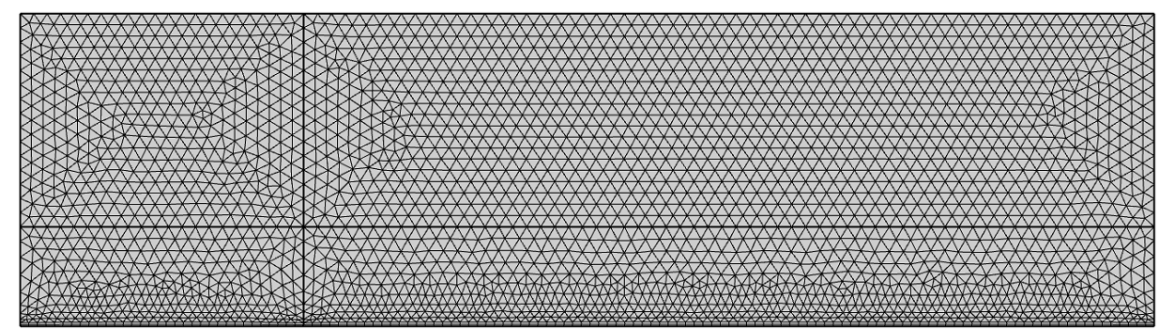

Figure 4: Computer-generated mesh for the model. Pictured mesh was automatically generated by COMSOL based on the applied physics on the "fine" resolution setting.

With the dilute species inlet being a constant concentration constraint and instantaneous outflow boundary conditions (no accumulation of the dilute species 
within the domain), the model domain reaches steady state after a "startup time" has elapsed. For a domain of this size, this time is about 1 second. This makes a time-independent study possible. Such simulations are useful as they take comparatively small times to compute and show long term trends in the data, most notably the diffusion profile.

COMSOL uses modules in the construction of models, which are systems containing all of the governing equations for a given phenomena written in terms that the software can freely couple with related modules. The two most important modules used in this simulation are "laminar flow" and "transport of a dilute species," which govern fluid advection and nanoparticle diffusion, respectively. Shown below are the governing equations used by COMSOL to simulate laminar flow:

$$
\begin{gathered}
\rho(u \cdot \nabla) u=\nabla \cdot(-\rho I+\tau) \\
\rho \nabla \cdot(u)=0 \\
\tau=\mu\left(\nabla u+(\nabla u)^{T}\right)
\end{gathered}
$$

where $\rho$ is the fluid density, $u$ is the fluid velocity, $\mu$ is the fluid viscosity, and $I$ and $\tau$ denote the identity tensor and Stokes's stress constitutive equation, respectively. These equations constitute the Navier Stokes equations for an incompressible fluid, and are subject to boundary conditions as specified above.

The governing equations used for dilute species transport by diffusion and convection are as follows:

$$
\begin{gathered}
\nabla \cdot J_{i}+u \cdot \nabla c_{i}=0 \\
J_{i}=-D_{i} \nabla c_{i}
\end{gathered}
$$

where $c_{i}$ is species concentration, $D_{i}$ is species diffusivity, and $u$ is again fluid velocity. These equations require a value for diffusivity to be input, which is given by eqn. (4). In the case where a chemical reaction is occurring to produce or consume the relevant species, an addition term, $R_{i}$, is added to expression, defined as the rate law for the reaction. Other assumptions used in these simulations include constant density for the two layers [12] and constant velocities for the clearance of the mucus layer and PCL [4, 25, 26]. Measurements of clearance rates are collected in vivo and thus already include the effects of gravity and breathing. The fiber volume fraction is for a healthy individual, within the ranges used by [20]. Mucin dimensions are within ranges reported by [27]. Table 1 includes the values of the constants provided to the model for the simulation.

\subsection{Model Equations}

For diffusivity of spherical particles in bulk solution, we use the Stokes-Einstein equation:

$$
D_{0}=\frac{k_{b} T}{6 \pi \mu r_{s}}
$$


Table 1: Collection of parameters used in calculations.

\begin{tabular}{|cccc|}
\hline Parameter & Definition & Value & Units \\
\hline$\rho$ & Density of fluid & 1000 & $\mathrm{~kg} / \mathrm{m}^{3}$ \\
$\mu$ & Viscosity of water at body temperature & $6.922 \times 10^{-4}$ & $\mathrm{~Pa} \cdot \mathrm{s}$ \\
$U_{m u c}$ & Mucus average velocity & 5 & $\mathrm{~mm} / \mathrm{min}$ (in x-direction) \\
$U_{p c l}$ & PCL average velocity & 2.4 & $\mathrm{~mm} / \mathrm{min}$ (in X-direction) \\
$C_{A i}$ & Applied solute concentration & 1000 & $\mathrm{~mol} / \mathrm{m}^{3}$ \\
$L_{p}$ & Depth of PCL & 7 & $\mu \mathrm{m}$ \\
$\phi$ & Fiber volume fraction & 0.0025 & unitless \\
$T$ & Body temperature & 310.15 & $\mathrm{~K}$ \\
$r_{f}$ & Mucin fiber radius & 5 & $\mathrm{~nm}$ \\
\hline
\end{tabular}

where $k_{b}$ is Boltzmann's constant, $T$ is absolute temperature, $\mu$ is dynamic viscosity of the pure solvent (in this case water), and $r_{s}$ is the Stokes radius of the solute particle. We allow $r_{s}$ to vary throughout our simulations. However, mucins provide a steric hindrance to diffusion which must be accounted for. The mucus is porous (average pore size around $150 \mathrm{~nm}[28,8]$ ), but the network is not rigid. It then follows that mucus is better described as a hydrogel, where diffusivity is a function of fiber concentration (rather than a function of pore size). We solve for an effective diffusivity, $D$, using a correlation from the literature that accounts for both steric and hydrodynamic interactions in a fibrous hydrogel [29]:

$$
\frac{D}{D_{0}}=\exp \left(-0.84 f^{1.09}\right) \exp \left(-a \phi^{b}\right)
$$

where

$$
f=\left(1+\frac{1}{\lambda}\right)^{2} \phi
$$

and

$$
\begin{aligned}
& a=3.727-2.460 \lambda+0.822 \lambda^{2} \\
& b=0.358+0.366 \lambda-0.0939 \lambda^{2}
\end{aligned}
$$

where $\lambda=r_{f} / r_{s}$ and $\phi$ is the fiber volume fraction $(0.0005 \leq \phi \leq 0.01$ in healthy individuals [20]). This equation is attractive, as it is fully characterized by only three variables: fiber radius $\left(r_{f}\right)$, particle radius $\left(r_{s}\right)$, and fiber volume fraction $(\phi)$. Particle radius would be one of the primary design variables for a study of either aerosolized drug efficacy or pathogenic infectivity (Fig. 5). Eqn. (4) does not assume a pore size or shape. Rather, hindrance is measured from the likelihood that a diffusing particle will collide with a fiber. Additionally, eqn. (4) does not rely on Brinkman or Effective Medium approximations. The Brinkman equation is a variation of Darcy's Law that is designed to describe flow in media where the grains of the media are themselves porous, and requires measurement of an effective viscosity. Effective Medium theory as applied to 
these situations, in short, considers the mucus to be characterized only by its Darcy permeability [30]. Both of these parameters are generally more difficult to calculate, measure, or estimate than $r_{s}, r_{f}$, and $\phi$. Thus, eqn. (4) is far more approachable than similar equations that rely on these approximations, but also tends to fit the data more accurately [29].

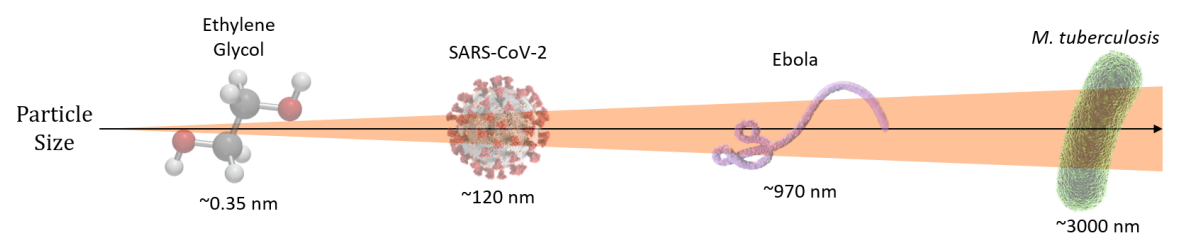

Figure 5: Examples of particles on the size scale used in these simulations. Larger particles are much more susceptible to steric hindrance in the mucus and require more time to successfully penetrate the mucus.

Considering the relation of mucus viscosity to shear rate [31], we chose to model the mucus layer as a Carreau fluid. The Carreau model is used in other applications to simulate similar biological fluids with non-Newtonian characteristics, like blood. The governing equation is given as:

$$
\mu_{e f f}(\dot{\gamma})=\mu_{i n f}+\left(\mu_{0}-\mu_{i n f}\right)\left(1+(\tau \dot{\gamma})^{2}\right)^{\frac{n-1}{2}}
$$

Parametrization of the data presented by [10] resulted in values of $\mu_{i n f}=0$ (assuming that viscosity is small at infinite shear so that this term drops out of the equation), $\mu_{0}=302.4894 \mathrm{~Pa} \cdot \mathrm{s}, \tau=8439.365 \mathrm{~s}$, and $n=0.37463$. These values were found via a nonlinear regression of those three variables using POLYMATH. The raw data was sourced from [10] and digitized using WebPlotDigitizer. The raw data is for cervicovaginal mucus, but [11] shows that all sources of mucus have very similar shear-thinning viscous behavior (with the exception of ovulatory cervicovaginal mucus, but the data in [10] is nonovulatory).

\section{Results and Discussion}

Before the diffusion of a dilute species is considered, it is important to verify that the fluid velocity profile is realistic. In the biological system, the mucus layer is so viscous (due to the tangled network of mucins) that it moves largely as a single sheet. The periciliary layer is more Newtonian and thus less susceptible to bulk movement and is cleared more slowly $[12,26,32]$. Due to these differences in behavior, the Carreau correlation described in eqn. (8) only applies to the upper mucus layer, with the viscosity of the PCL approximated as water. Fig. 6 shows this bulk movement of the mucus layer, as well as the rapid drop in velocity in the PCL due to both the no-slip condition with the epithelium and its differing properties. 


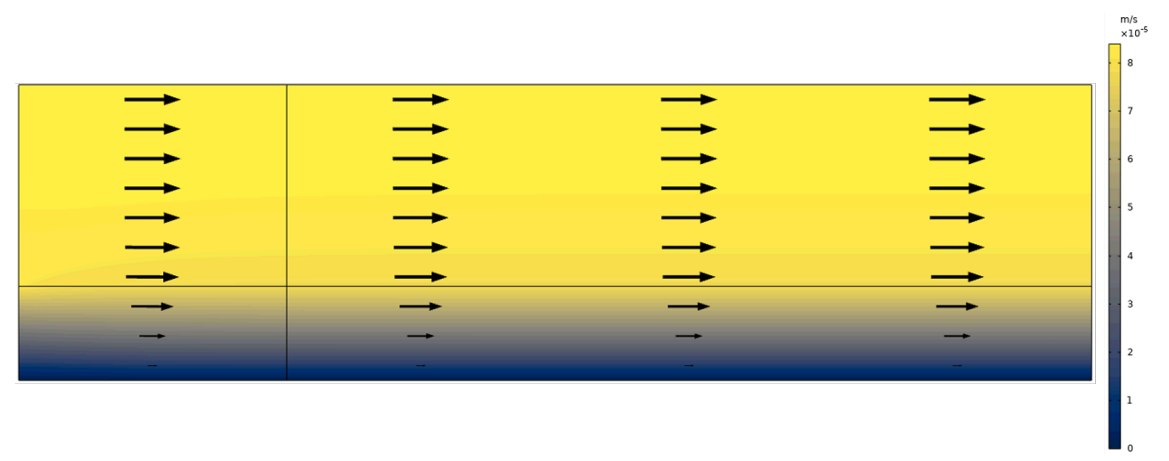

Figure 6: Velocity profile given by the model. Note a nearly constant velocity throughout the mucus (upper) layer. $U_{m u c}$ in the x-direction is specified as 5 $\mathrm{mm} / \mathrm{min}$, an average in vivo tracheal mucus clearance rate $[4,25]$ that is listed in Table 1.

Concurrent with this velocity profile arising from the viscous nature of the two fluids, it follows that the periciliary layer is subject to a high shear rate, as cilia beat through it constantly. Conversely, the mucus layer is subject to a low shear rate due to its elasticity. In short, the shear rate is another measure of the mucus moving as a sheet, as the bulk mucus moves in response to cilial beating. The periciliary layer is also responsive to this beating, but to a much smaller extent due to both the no-slip condition for the velocity profile and the much lower viscosity of the PCL. Another way of interpreting Fig. 7 is as a measure of resistance to movement. High shear rate in the PCL is indicative of inertial stresses that keep it from clearing at the same rate as the mucus. Similar to the velocity profile, the values are nearly constant in the mucus but subject to a gradient in the PCL, albeit numerically inverted when compared to the velocity profile.

Once a velocity field has been constructed, a "drug" may be applied to see its diffusive properties. All simulations in this paper used a constant delivery concentration of $1000 \mathrm{~mol} / \mathrm{m}^{3}$, or $1 \mathrm{~mol} / \mathrm{L}$. In the delivery of an actual drug delivery would not be constant, but all mucus within the domain has a space time of about 1 second due to MCC, so this was considered a short enough timescale for constant delivery to be valid. It is assumed that this concentration is sufficiently dilute that the delivered drug does not significantly change the volume of the system. COMSOL natively supports parameter sweeps, where a single solution of the system evaluates a range of parameters. This functionality was first utilized to compare the effect of mucus layer depth $\left(L_{m}\right)$ on drug penetration. Shown in Fig. 8 are the concentration for profiles for three different mucus depths, representing delivery to the bronchioles, bronchus, and trachea, respectively. Drug particle radius was held constant at $20 \mathrm{~nm}$.

Parameter sweeps for drug particle radius were also performed (Fig. 9). In this case, mucus depth was held constant at $15 \mu \mathrm{m}$. Concentration profiles for 
bioRxiv preprint doi: https://doi.org/10.1101/2021.10.18.464809; this version posted October 19, 2021. The copyright holder for this preprint (which was not certified by peer review) is the author/funder. All rights reserved. No reuse allowed without permission.

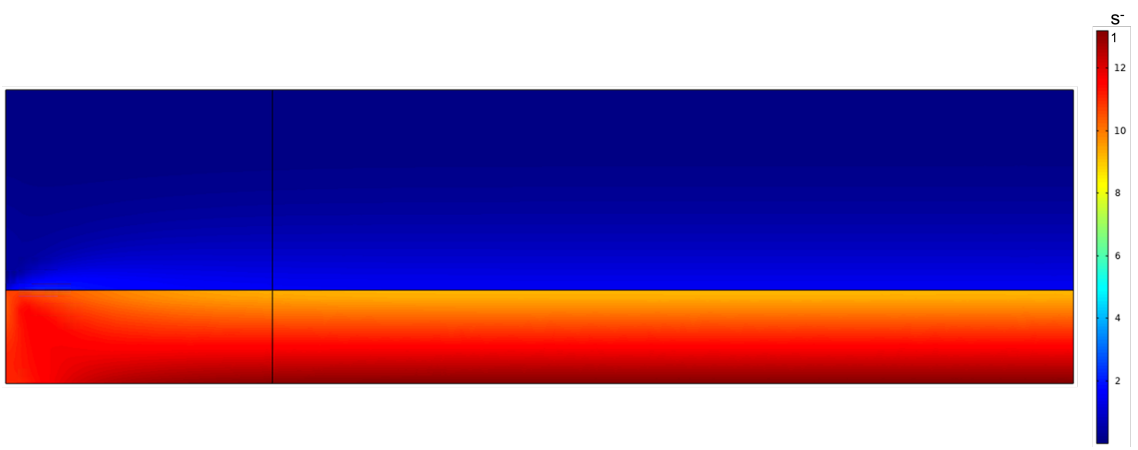

Figure 7: Shear rate in the mucus (upper) and periciliary (lower) layers. The mucus (upper) layer has a comparatively small and constant shear rate due to its elasticity.

particles of radii $5 \mathrm{~nm}$ and $60 \mathrm{~nm}$ are shown, displaying the strong dependence of particle size on effective diffusivity. The profile for a $20 \mathrm{~nm}$ particle under these conditions is identical to the second profile in Fig. 8.

As expected, thicker mucus layers are more effective barriers to particle transport than thinner layers, and larger particles have lower diffusivities than smaller particles. This is generally true for any passive diffusion in any fluid, but is particularly relevant here as drug that does not fully cross both fluid layers is ultimately removed from the lungs by mucociliary clearance and eliminated. It is clear that particles deposited deeper in the lungs will have greater success penetrating the mucus and reaching tissue (higher bioavailability) than particles deposited higher in the lungs. However, this answer cannot be exhaustive, as certain patients may benefit from a specific localized dosage or tissue targeting, such as in cancer treatment [2]. For treatment regimes such as these, it is important to note that an appreciable amount of the applied drug only reaches the epithelium some distance upstream of the dosage site.

For any particle in any mucus depth, there is some distance upstream where delivered dosage is maximized. By adding a cut line at the epithelial surface and exporting the concentration results along the $y=0$ line, this distance can be found (Fig. 10). Another important trend to notice is that both increased particle size and increased mucus depth result in "flattening" of the curves shown in Figs. 10 and 11. Increases to either of these parameters results in the maximum occurring further downstream and being smaller in magnitude. When a treatment is designed, a dosage may be selected such that the desired concentration to achieve a physiological response is achieved at the maximum, and a dosage site selected based on the distance required to reach this peak. The model provides information regarding the magnitude of dosage delivered along the lung epithelium. However, factors that may optimize delivery such as shrinking the particle size or delivering deeper into the lungs may not be viable. Particle size causes variations in impaction with the mucus when inhaled [33], and a particle 


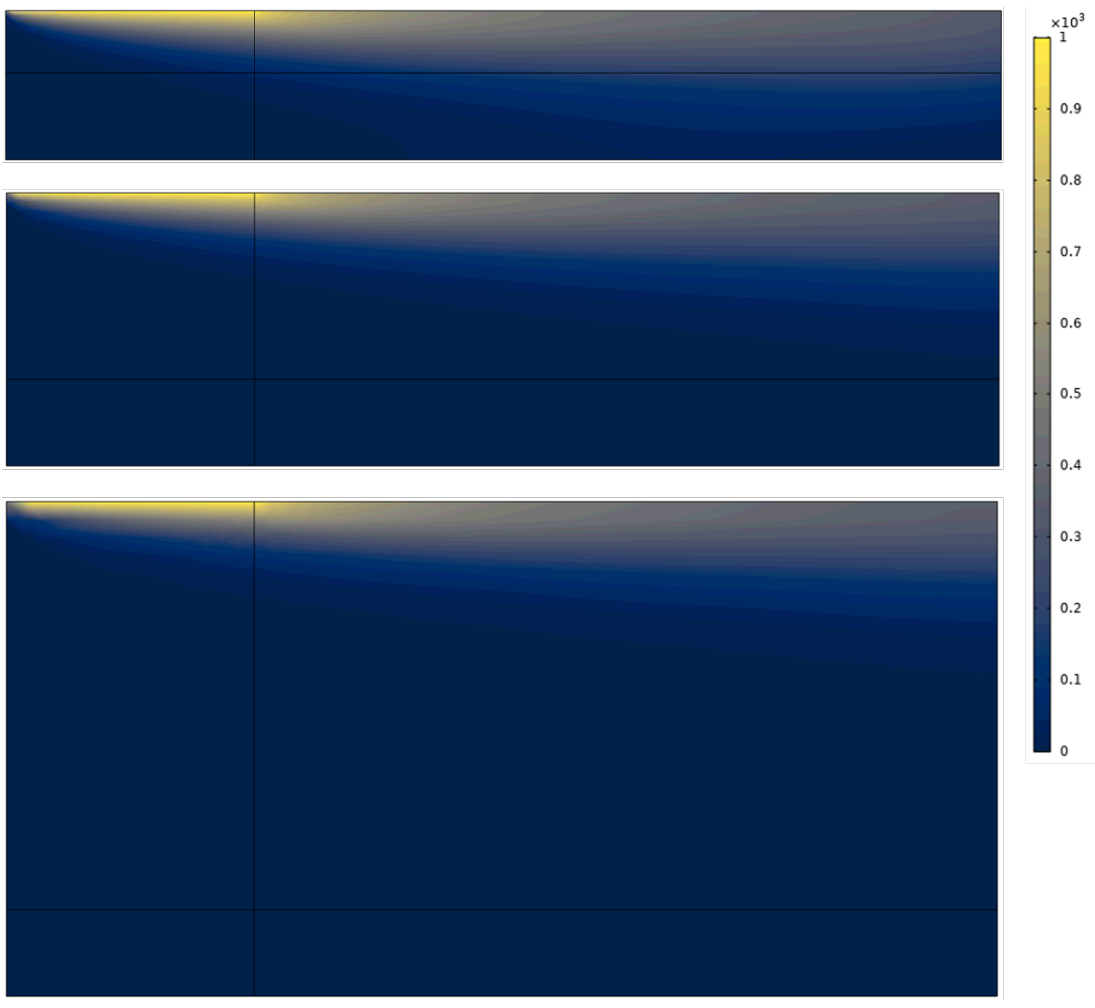

Figure 8: Diffusion profile for a particle with a radius of $20 \mathrm{~nm}$ for various mucus depths $\left(5 \mu \mathrm{m}, 15 \mu \mathrm{m}\right.$, and $33 \mu \mathrm{m}$, from top to bottom). Units are in $\mathrm{mol} / \mathrm{m}^{3}$. For a particle of any size, the drug would need to be administered deeper into the lungs than the targeted site to ensure a maximum amount of dosage reaches that area. However, location of the targeted site also determines how much deeper, as less distal lung regions have thicker mucus layers, which take longer to cross.

may require a protective coating to prevent side reactions, denaturation, or electrostatic entrapment $[34,35,36,37]$. It has been hypothesized that drugs may be applied that slow the beating of cilia, therefore decreasing mucus clearance and giving the drug more time to cross the fluid layers. This is an interesting idea, but many pathogenic diseases of the lung are directly caused by MCC dysfunction, so this method would likely increase the risk of complications [38]. In diseases characterized by MCC dysfunction, such as cystic fibrosis, asthma, and COPD, steric (and electrostatic) hindrance is often highly accentuated due to higher-than-normal mucin concentrations. In these diseases, additional drugs (or even simply water) might be applied to cause the mucus to behave more like the healthy case and improve outcomes [39].

One major limitation of the model is the assumption that electrostatic in- 


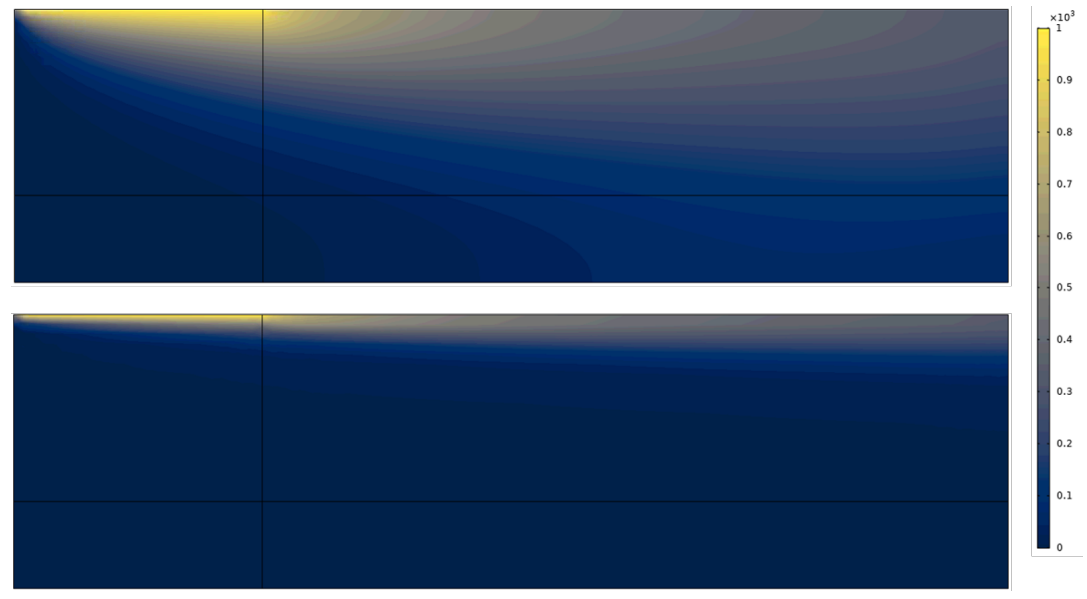

Figure 9: Diffusion profile for a particle with a radius of $5 \mathrm{~nm}$ (top) and a particle with a radius of $60 \mathrm{~nm}$ (bottom) with a mucus depth of $15 \mu \mathrm{m}$. Units are in $\mathrm{mol} / \mathrm{m}^{3}$. As expected, smaller particles diffuse faster than larger particles.

teractions are negligible. This assumption is valid for many particles including many viruses and polyethylene glycol-coated nanoparticles, but certainly not all particles. Mucins are anionic and lipophilic, so cationic or lipophilic particles are subject to electrostatic hindrance, which can be immobilizing [20]. Repulsive anionic interactions between particles and mucins result in a sort of channel flow, which is less hindering but still causes an effective shrinkage of pores. Particles that are surface neutral but hydrophilic (either polar or zwitterionic) typically are the most successful at penetrating mucus.

Some simulations have also been made for the case of chemical reactions occurring in solution. COMSOL natively supports this, although reaction rate laws must be provided. Unwanted side reactions would undoubtedly be an issue in many aerosolized drug applications, but one specific application is the simulation of prophylactics in the mucus. Lung mucosa, like other mucosal tissues, is an immunoactive region, with antibodies present in solution. When these antibodies bind to an antigen, the resulting complex is often trapped sterically or electrostatically. Using these relationships, it can be shown whether a given level of antibody expression in the mucus is sufficient to prevent infection (defined as a certain amount of antigen reaching the epithelium). In these simulations, the diffusing particle is not a drug but a disease-causing particle. In fact, the model is equally capable of simulating the penetration of pathogens through the mucus as it is man-made nanoparticles. 


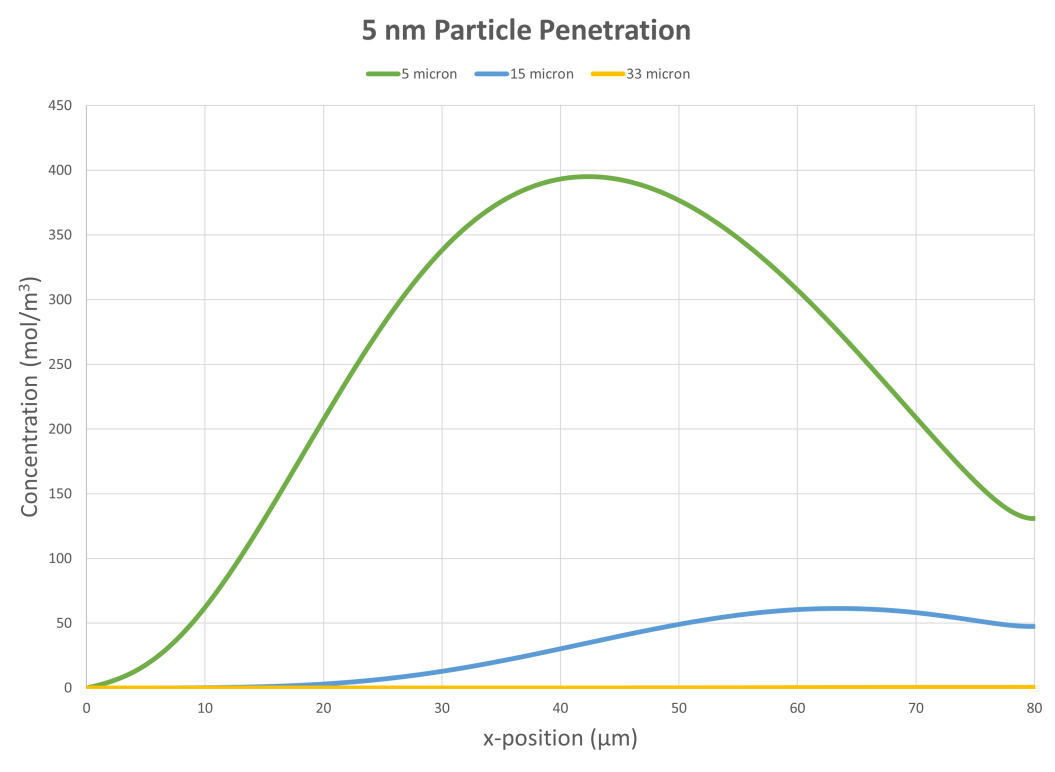

Figure 10: Concentration at the epithelial surface as a function of position in the steady-state model. This simulation is for a particle with $\mathrm{r}_{\mathrm{s}}=5 \mathrm{~nm}$, where each curve indicates a different depth of the mucus layer $\left(L_{m}\right)$. This small particle reaches the epithelium quite close to the inlet (or delivery site), whereas in thicker mucus it reaches the epithelium further from the inlet and has a maximum smaller in magnitude. The $\mathrm{x}$-position of the maximum of this curve could be an ideal delivery site, with the upstream dosage site selected specifically to reach this point.

\section{Conclusions}

The model developed using COMSOL Multiphysics returns a biologically realistic simulation of the mucociliary clearance mechanism. The model is customizable to the needs of the modeler or even the physiology of a patient, including both mucus properties and physical dimensions of the simulation domain. The administered particle is likewise customizable, although the model only accounts for steric and hydrodynamic hindrance. Existing simulations accounting for electrostatic interactions in "interacting gels" like mucus are computationally intense [22], and a macroscopic mathematical relationship that may be added to eqn. (4) is needed. By exporting transport simulation results, plots of concentration reaching the epithelium versus distance downstream from the dosage site may be created. Using this information the optimal dosage site may be identified, which is relevant in the development of aerosolized drug treatments for localized diseased tissues, including tumors. One possible future refinement is replacing the concentration inlet with a time- and position-dependent step function, simplifying the domain to only an upper mucus layer and lower PCL 


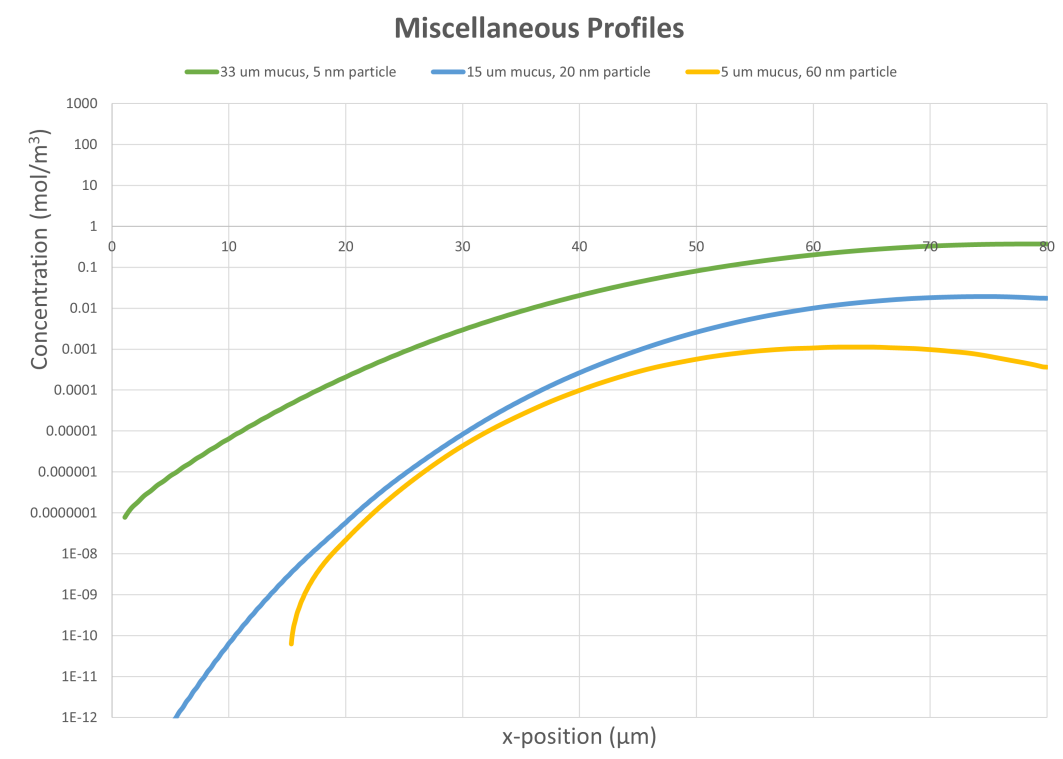

Figure 11: Concentration at the epithelial surface as a function of position in the steady-state model, with concentration plotted on a logarithmic scale. These curves are for three separate combinations of particle size $\left(r_{s}\right)$ and mucus depth $\left(L_{m}\right)$. Increases in either of these parameters result in the penetration maximum being smaller in magnitude and occurring further from the inlet.

and adding possibilities for non-steady-state simulations. 


\section{References}

[1] Y. Feng, X. Chen, and M. Yang. An In Silico Investigation of a LobeSpecific Targeted Pulmonary Drug Delivery Method. Proc 2018 Design Med Devices Conf, 34:1-4, 2018.

[2] E. L. Kolewe, Y. Feng, and C. A. Fromen. Realizing Lobe-Specific Aerosol Targeting in a 3D-Printed In Vitro Lung Model. J Aerosol Med Pulm Drug Deliv, 34:42-56, 2021.

[3] J. V. Fahy and B. F. Dickey. Airway Mucus Function and Dysfunction. N Engl J Med, 363:2233-2247, 2010.

[4] F. Taherali, F. Varum, and A. W. Basit. A Slippery Slope: On the Origin, Role and Physiology of Mucus. Adv Drug Delivery Rev, 124:16-33, 2018.

[5] S. Chateau, U. D'Ortana, S. Poncet, and J. Favier. Transport and Mixing Induced by Beating Cilia in Human Airways. Front Physiol, 9:161, 2018.

[6] R. A. Cone. Chapter 4: Mucus. In J. Mestecky, M. E. Lamm, P. Ogra, W. Strober, J. Bienenstock, J. McGhee, and L. Mayer, editors, Mucosal Immunology, pages 49-72. Academic Press, Cambridge, MA, 3rd edition, 2005.

[7] X. Murgia, B. Loretz, O. Hartwig, M. Hittinger, and C. Lehr. The Role of Mucus on Drug Transport and its Potential to Affect Therapeutic Outcomes. Adv Drug Delivery Rev, 124:82-97, 2018.

[8] B. Button, L. Cai, C. Ehre, M. Kesimer, D. B. Hill, J. K. Sheehan, R. C. Boucher, and M. Rubinstein. A Periciliary Brush Promotes the Lung Health by Separating the Mucus Layer from Airway Epithelia. Science, 337:937-941, 2012.

[9] S. W. Clarke and D. Pavia. Lung Mucus Production and Mucociliary Clearance: Methods of Assessment. Br J Clin Pharmac, 9:537-546, 1980.

[10] S. K. Lai, D. E. O'Hanlon, S. Harrold, S. T. Man, Y. Wang, R. Cone, and J. Hanes. Rapid Transport of Large Polymeric Nanoparticles in Fresh Undiluted Human Mucus. Proc Natl Acad Sci USA, 104:1482-1487, 2007.

[11] R. A. Cone. Barrier Properties of Mucus. Adv Drug Delivery Rev, 61:75-85, 2008.

[12] M. M. Norton, R. J. Robinson, and S. J. Weinstein. Model of Mucociliary Clearance and the Role of Mucus Rheology. Phys Rev E, 83:011921, 2011.

[13] G. A. Duncan, J. Jung, J. Hanes, and J. S. Suk. The Mucus Barrier to Inhaled Gene Therapy. Mol Ther, 24:2043-2053, 2016.

[14] M. I. Lethem. The Role of Tracheobronchial Mucus in Drug Administration to the Airways. Adv Drug Delivery Rev, 11:271-298, 1993. 
[15] R. K. Wolff. Effects of Airborne Pollutants on Mucociliary Clearance. Environ Health Perspect, 66:223-237, 1986.

[16] D. B. Yeates, N. Aspin, H. Levison, M. T. Jones, and A. C. Bryan. Mucociliary Tracheal Transport Rates in Man. J Appl Physiol, 39:487-495, 1975.

[17] B. C. Tang, M. Dawson, S. K. Lai, Y. Wang, J. S. Suk, M. Yang, P. Zeitlin, M. P. Boyle, J. Fu, and J. Hanes. Biodegradable Polymer Nanoparticles that Rapidly Penetrate the Human Mucus Barrier. Proc Natl Acad Sci USA, 106:19268-19273, 2009.

[18] Y. Feng, X. Chen, M. Yang, and K. Dong. Multiscale Computational Models for Respiratory Aerosol Dynamics with Medical Applications. Comput Math Methods Med, 2019:1-2, 2019.

[19] D. J. Smith, E. A. Gaffney, and J. R. Blake. Modelling Mucociliary Clearance. Respir Physiol Neurobiol, 163:178-188, 2008.

[20] J. Hansing and R. R. Netz. Particle Trapping Mechanisms Are Different in Spatially Ordered and Disordered Interacting Gels. Biophys J, 114:2653$2664,2018$.

[21] Y Cu and M. Saltzman. Mathematical Modeling of Molecular Diffusion through Mucus. Adv Drug Delivery Rev, 61:101-114, 2009.

[22] J. Hansing and R. R. Netz. Hydrodynamic Effects on Particle Diffusion in Polymeric Hydrogels with Steric and Electrostatic Particle-Gel Interactions. Macromolecules, 51:7608-7620, 2018.

[23] J. Kirch, M. Guenther, N. Doshi, U. F. Schaefer, M. Schneider, S. Mitragotri, and C. Lehr. Mucociliary Clearance of Micro- and Nanoparticles is Independent of Size, Shape, and Charge-an ex vivo and in silico Approach. J Controlled Release, 159:128-134, 2012.

[24] L. B. Sims, H. A. Miller, M. E. Halwes, J. M. Steinbach-Rankins, and H. B. Frieboes. Modeling of Nanoparticle Transport Through the Female Reproductive Tract for the Treatment of Infectious Diseases. Eur J Pharm Biopharm, 138:37-47, 2019.

[25] H. K. Shete, S. S. Vyas, V. B. Patravale, and J. I. Disouza. Pulmonary Multifunctional Nano-Oncological Modules for Lung Cancer Treatment and Prevention. J Biomed Nanotechnol, 10:1863-1893, 2014.

[26] H. Matsui, S. H. Randell, S. W. Peretti, C. W. Davis, and R. C. Boucher. Coordinated Clearance of Periciliary Liquid and Mucus from Airway Surfaces. J Clin Invest, 102:1125-1131, 1998.

[27] S. K. Lai, Y. Wang, D. Wirtz, and J. Hanes. Micro- and Macrorheology of Mucus. Adv Drug Delivery Rev, 61:86-100, 2009. 
[28] N. Kim, G. A. Duncan, J. Hanes, and J. S. Suk. Barriers to Inhaled Gene Therapy of Obstructive Lung Diseases: A Review. J Controlled Release, 240:465-488, 2016.

[29] R. J. Philips. A Hydrodynamic Model for Hindered Diffusion of Proteins and Micelles in Hydrogels. Biophys J, 79:3350-3354, 2000.

[30] E. M. Johnson, D. A. Berk, R. K. Jain, and W. M. Deen. Hindered Diffusion in Agarose Gels: Test of Effective Medium Model. Biophys J, 70:1017-1026, 1996.

[31] J. R. Vélez-Cordero and E. Lauga. Waving Transport and Propulsion in a Generalized Newtonian Fluid. J Non-Newtonian Fluid Mech, 199:37-50, 2013.

[32] J. Hussong, R. Lindken, P. Faulhammer, K. Noreikat, K. V. Sharp, W. Kummer, and J. Westerweel. Cilia-Driven Particle and Fluid Transport Over Mucus-Free Mice Tracheae. J Biomech, 46:593-598, 2013.

[33] P. M. Schlosser, B. A. Asgharian, and M. Medinsky. Inhalation Exposure and Absorption of Toxicants. In C. A. McQueen, editor, Comprehensive Toxicology, pages 75-109. Elsevier Science, New York, NY, 2nd edition, 2010.

[34] M. E. Halwes, K. M. Tyo, J. M. Steinbach-Rankins, and H. B. Frieboes. Computational Modeling of Antiviral Drug Diffusion from Poly(lactic-coglycolic-acid) Fibers and Multicompartment Pharmacokinetics for Application to the Female Reproductive Tract. Mol Pharmaceutics, 15:1534-1547, 2018.

[35] G. Osman, J. Rodriguez, S. Y. Chan, J. Chisholm, G. Duncan, N. Kim, A. L. Tatler, K. M. Shakesheff, J. Hanes, J. S. Suk, and J. E. Dixon. PEGylated Enhanced Cell Penetrating Peptide Nanoparticles for Lung Gene Therapy. J Controlled Release, 285:35-45, 2018.

[36] H. P. Patil, D. Freches, L. Karmani, G. A. Duncan, B. Ucakar, J. S. Suk, J. Hanes, B. Gallez, and R. Vanbever. Fate of PEGylated Antibody Fragments Following Delivery to the Lungs: Influence of Delivery Site, PEG Size and Lung Inflammation. J Controlled Release, 272:62-71, 2018.

[37] X. Huang, J. Chrisholm, J. Zhuang, Y. Xiao, G. Duncan, X. Chen, J. S. Suk, and J. Hanes. Protein Nanocages that Penetrate Airway Mucus and Tumor Tissue. Proc Natl Acad Sci USA, 114:E6595-E6602, 2017.

[38] J. C. Nawroth, A. M. van der Does, A. Ryan (Firth), and E. Kanso. Multiscale Mechanics of Mucociliary Clearance in the Lung. Phil Trans $R$ Soc, 375:1-8, 2019. 
[39] N. Nafee, K. Forier, K. Braeckmans, and M. Schneider. Mucus-Penetrating Solid Lipid Nanoparticles for the Treatment of Cystic Fibrosis: Proof of Concept, Challenges and Pitfalls. Eur J Pharm Biopharm, 124:125-137, 2018. 